\title{
Sowjetische Beiträge zur Faserforschung und Textiltechnik
}

in deutscher Übersetzung

Herausgegeben vom Institut für Polymerenchemie in Teltow-Seehof, BURKART PHILIPP

und dem Institut für Technologie der Fasern in Dresden, WOLFGANG BOBETH

der Akademie der Wissenschafen der DDR

Je Heft 56 S. $-21 \times 29,7 \mathrm{~cm}-$ jährlich erscheinen 12 Hefte - je Heft 7,50 M

Bestell-Nr. 1074

Aus zunächst 4 sowjetischen Fachzeitschriften bringt die Zeitschrift ausgewählte Beiträge in deutscher Übersetzung und vermittelt zugleich einen Überblick über den Inhalt der neuesten Hefte.

In der Sowjetunion erscheinen in zunehmendem Maße wissenschaftliche und technische Veröffentlichungen, deren eingehende Kenntnis für die Mitarbeiter der Chemiefaser- und Textilbetriebe sowie der einschlägigen Forschungsstellen dringend erforderlich ist. Die wichtigsten dieser Arbeiten auch den mit der russischen Sprache nicht ausreichend vertrauten Fachleuten zu vermitteln, ist Aufgabe dieser Zeitschrift. Bei der Themenauswahl werden die theoretischen und die praktischen Fragen in gleichem Umfang berücksichtigt. Ausgewertet werden in erster Linie folgende Zeitschriften:

Vysokomolekuljarnye Soedinenija (Hochmolekulare Verbindungen),

Chimičeskie Volokna (Chemische Fasern),

Izvestija vysšich učebnich Zavedenij Technologija tekstil'noj Promyslennosti (Hochschulnachrichten, Technologie der Textilindustrie),

Tekstil'naja Promyšlennost (Textil-Industrie).

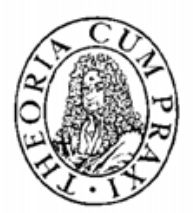

Bestellungen durch eine Buchhandlung erbeten

A K A D E M I E - V E R L A G B E R L I N

Die Zcitschrift ,„Faserforschung und Textiltechnik“" erscheint monatlich in Heften zu 48 Textseiten im Format A 4. Der Preis für das Einzelheft beträgt

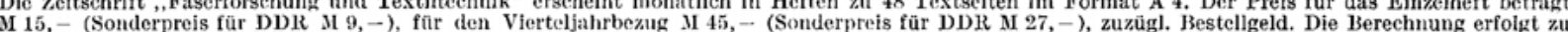
Beginn eines Vierteljahrs fūr 3 Hefte. Bestellumgen aus dem (iebiet der Deutschen Demokratischen lkepublik an ein Postamt, eine l3uchhandlung oder den Verlag, aus der Deutschen Bundesrepublik an eine Buchhandlung oder die Auslieferungsstelle Kunst und Wissen, Brich Bieber, 7 Stuttgart 1, Wilhelmstraße 4-6, aus dem Ausland an eine Importbuchhandlung, den Deutschen J3uch-Wxport und -Import GmbH., 701 Jeipzig, Postschließfach 276, oder den Akademie-Verlag GmbH., 108 Berlin, Leipziger Str. 3 -4 (Ferniruf: 220441; 'Telex-Nr. 0112020; Postscheckkonto 35021) erbeten. Bestellnummer dieses Heftes: 1014/2t/4. Alleinige Anzeigenamalame J)WAG-WJRB NG, 1054 Berlin, Wilhelm-Pieck-Str. 49, und alle DEWAG-Betriebe in den Bezirksstädten der DDR. - Jestellungen in der CdSSR nehmen entgegen: Städtische Abteilungen von, ,SOJUZPECHATJ“ bzw. Postämter und Postkontore.

Herausgeber und verantwortlich für den Inhalt: Prof. Dr. Dr. lirich Correns, Institut für Polymerenchemie der Akademie der Wissenschaften der DDR, 153 Teltow-Sechof, Fernruf: Teltow 4831; Prof. 1)r.-Ing. habil. Wolfgang Bobeth, Institut für Technologie der Fasern der Akademie der Wissenschaften der DDR, 801 Dresden, Hohe Str. 6, Fernruf: 44721 ; Prof. Dr.-Ing. Hans Böhringer, 682 Rudolstadt; Prof. Dr. Hermann Klare, Prof. Dr. habil. Burkart Philipp und Dr. habil. Christian Ruscher, Institut für Polymerenchemie der Akademie der Wissenschaften der DDR, 153 Teltow-Seehof, Fernruf: Teltow 4831. Straße 3-4. Satz und Druck: Druckhaus , Maxim Gorki", 74 Altenburg. ‥ Veröffentlicht unter der Lizenznummer 1280 des Presseantes beim Vorsitzenden des Ministerrates der Deutschen Demokratischen Republik.

Manuskriptsendungen sind an einen der Herausgeber oder die Schriftleitung zu richten. Für Inhalt und Form gelten die , Richtlinien für die Annahme und Abfassungen von Beiträgen ", erhältlich von der Schriftleitung. J)ie Verfasser größßerer wissenschaftlicher Arbeiten erhalten außer den Honorar ein Heft und 50 Sonderdrueke ihrer Arbeit unentgeitlich. Nachdrucke sowie Ubersetzungen in fremde Sprachen des Inhalts dieser Zeitschrift und deren Verbreitung - auch auszugsweise mit Quellenaugabe - bedürfen der schriftlichen Vereinbarung mit dem Verlag. 